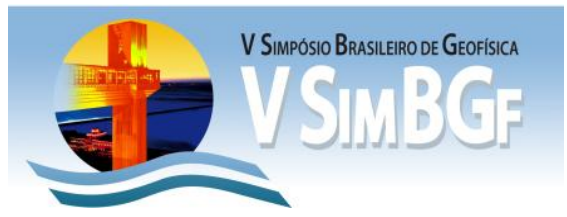

\title{
ANÁLISE DE TELESSISMOS ANDINOS REGISTRADOS NA ESTAÇÃO SISMOGRÁFICA DE AQUIDAUANA - MS (AQDB)
}

Franciane Rodrigues, Centro de Ciências Exatas e Tecnológicas/UFMS.

Leonardo Demétrio de Freitas Felício, Centro de Ciências Exatas e Tecnológicas/UFMS.

Fábio L. Dias, Instituto de Astronomia, Geofísica e Ciências Atmosféricas /USP

Marcelo S. Assumpção, Instituto de Astronomia, Geofísica e Ciências Atmosféricas /USP.

Prof. Dra . Edna Maria Facincani, Campus de Aquidauana/UFMS.

Prof. Dr. Hamilton Perez Soares Corrêa, Centro de Ciências Exatas e Tecnológicas/UFMS.

Copyright 2012, SBGf - Sociedade Brasileira de Geofísica

Este texto foi preparado para a apresentação no V Simpósio Brasileiro de Geofísica, Salvador, 27 a 29 de novembro de 2012. Seu conteúdo foi revisado pelo Comitê Técnico do V SimBGf, mas não necessariamente representa a opinião da SBGf ou de seus associados. É proibida a reprodução total ou parcial deste material para propósitos comerciais sem prévia autorização da SBGf.

\section{Resumo}

Este trabalho tem por objetivo realizar o levantamento de atividades sísmicas vindos da região andina a uma distância acima de $1500 \mathrm{~km}$ (telessismos), registrados na Estação Sismográfica de Aquidauana - MS (AQDB). Foram analisados 10 eventos ocorridos no período de 1 은 de agosto a 31 de dezembro de 2011, com o auxilio dos Programas TAUP e SAC (SEISMIC ANALYSIS CODE). Foram feitos uma tabela com características gerais dos sismos ocorridos e gráficos, estes mostrando a correlação entre as magnitudes $m_{b}$ e $M_{s}$ calculadas em relação ao NEIC, possibilitando uma compreensão das atividades sísmicas das placas de Nazca e Sulamericana.

\section{Introdução}

O avanço no estudo da Sismologia e a implantação das redes sismográficas do Projeto Rede Sismográfica Integrada do Brasil - BRASIS (BRAzilian Seismographic Integrated Systems) têm contribuído no monitoramento sísmico e telessísmico do território brasileiro, e consequentemente na América do Sul. Os telessismos ocorrem em outras regiões da Terra, liberando energias de forma significante e com magnitudes que podem ser registradas por estações sismográficas a milhares de quilômetros de distância do epicentro. Eles são usados para mapear as variações da espessura da litosfera/crosta, permitindo um melhor entendimento da estrutura e evolução geológica do embasamento cristalino, que por sua vez, influenciam a subsidência de bacias sedimentares. A primeira estação sismográfica permanente de Mato Grosso do Sul, a Estação Sismográfica de Aquidauana (AQDB) foi inaugurada no dia $1^{\circ}$ de agosto de 2011 e localizada no Campo de Instrução do 9 Batalhão de Engenharia de Combate (9응 BEC), distante nove quilômetros da cidade de Aquidauana - MS. Este trabalho tem por objetivo realizar o levantamento de atividades sísmicas vindos da região andina a uma distância acima de 1500 km (telessismos), registrados na Estação Sismográfica de Aquidauana
(AQDB) no segundo semestre de 2011, contribuindo com a BRASIS.

\section{Metodologia}

Os sismogramas correspondentes aos eventos telessismicos foram analisados através do Programa SAC (SEISMIC ANALYSIS CODE) e os tempos de chegada das ondas $\mathrm{P}$ e $\mathrm{S}$ foram obtidos no TAUP. Esses parâmetros possibilitaram o cálculo das magnitudes $m_{b}$ (Magnitude de onda $P$ ) e $M_{s}$ (Magnitude de onda superficial), baseada em ondas superficiais. As magnitudes desses telessismos estão divulgadas no catálogo do NEIC (National Earthquake Information Center) e os eventos sísmicos coletados em AQDB foram calculados e comparados com eventos num instante anterior ao registrado.

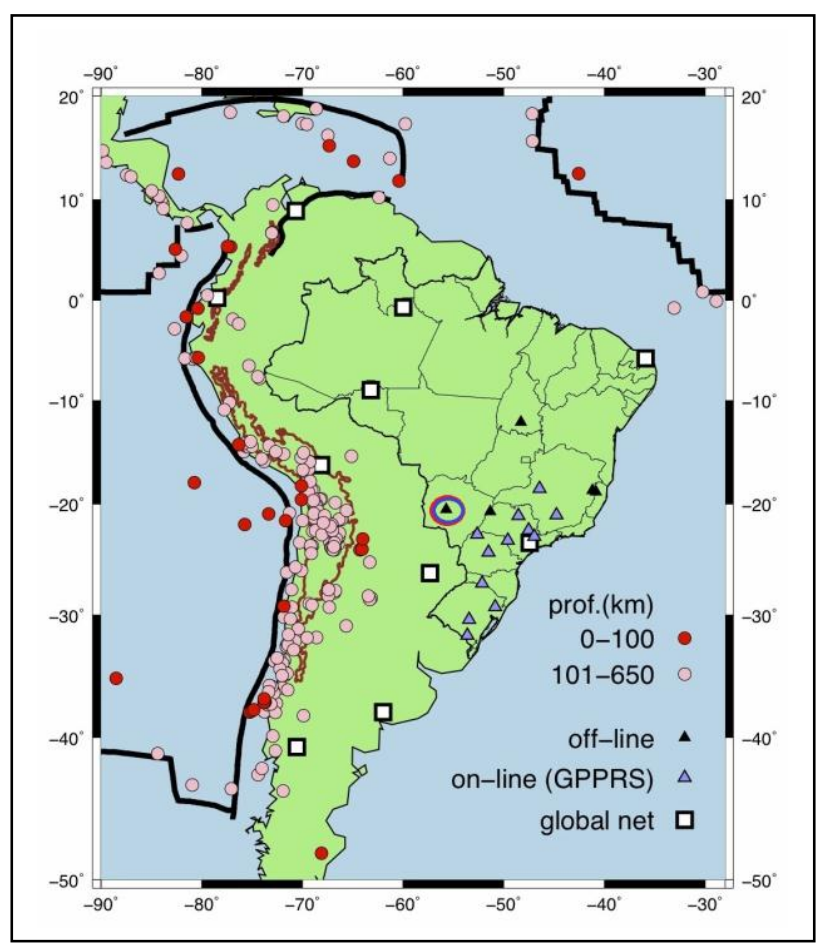

Figura 1 - Localização da Estação Sismográfica $A Q D B$ indicada no círculo (-20 47' $58^{\circ}$ ' $S$; $-55^{\circ} 69^{\prime}$ 97' $W$ ) e de sismos entre as profundidades: 0-100 (vermelho), 101-650 (rosa) e as estações: on/off-line e global net, determinados automaticamente pelo sistema SC3 do IAG. As profundidades tem baixa precisão 


\section{Resultados}

América do Sul está localizada na região de interface entre duas grandes placas tectônicas: Sul-americana e Nazca. Seu limite de interação na porção oeste é marcado pelo processo de subducção, possibilitando a presença de forte atividade sísmica. A Estação Sismográfica de Aquidauana (AQDB), localiza-se na latitude -20 47' 58" S e longitude -55 69' 97" W, no município de Aquidauana, no Estado de Mato Grosso do Sul (MS). Foram analisados 10 eventos ocorridos no período de $1^{\circ}$ de agosto a 31 de dezembro de 2011. Para cada sismograma, os parâmetros analisados e calculados foram: data, dia Juliano, tempo de origem do evento, latitude, longitude, magnitudes Ms e $m_{b}$ (AQDB e NEIC), profundidade $(\mathrm{Km})$, distância $(\mathrm{Km})$ e epicentro (Figura 2 e 3 ).

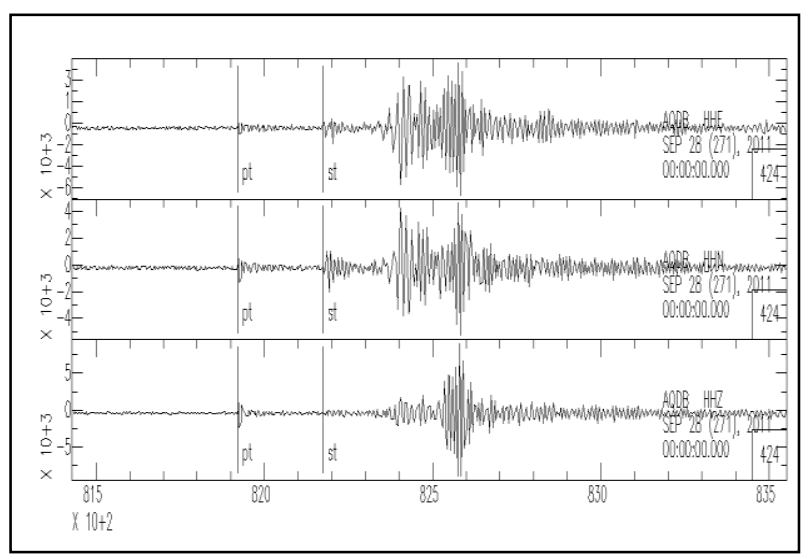

Figura 2 - Sismo do dia 28/09/2011 (271) com marcações teóricas das ondas $P$ e $S$ $(22: 45: 21 ; 22: 53: 35)$.

\section{Discussão e Conclusões}

Os telessismos andinos identificados na AQDB possuem seus epicentros variando entre $1.148 \mathrm{~km}$ a $3.173 \mathrm{~km}$ de distância até a Estação, sendo $80 \%$ do Chile, $10 \%$ da Argentina e $10 \%$ do Peru (Figura 3 e 4).

\begin{tabular}{|c|c|c|c|c|c|c|c|}
\hline \multicolumn{8}{|c|}{ Nimero de erentos sismicos: 10} \\
\hline Data (2011) & Dia Juliano & Tempo de origem & Latitude & Longithde & Profundidade $\left(\mathrm{K}_{\mathrm{m}}\right]$ & Distância $(\mathrm{Km})$ & Epicentro \\
\hline 248 & 236 & 17:46:11 & $.7,641$ & $.74,25$ & 147 & 2775 & Borda Pen - Chle \\
\hline 288 & 240 & $0+3032$ & $.72,296$ & $-332,214$ & 25,1 & 2169 & Costa Cerrial do Chlite \\
\hline 29 & 245 & 13:47:09 & $.28,398$ & $-63,029$ & 578,9 & 1148 & Sartiago Dat Estreo Argetina \\
\hline 39 & 246 & $1620: 41$ & $-38,435$ & $.74,907$ & 12 & 2714 & Costa Cerrad do Chile \\
\hline 79 & 250 & $11: 21: 50$ & $-33,300$ & $.72,170$ & 19:2 & 2161 & Costa Cestral do Chite \\
\hline 149 & 257 & 070.0351 & $-32,696$ & $.71,797$ & 37 & 2111 & Costa Cerrald do Chille \\
\hline 289 & 271 & 22:-1:12 & $.379,95$ & $.73,853$ & 10 & 2608 & Costa Certial do Chite \\
\hline 3010 & 303 & $1853: 43$ & $.25,741$ & $.70,959$ & 47 & 1658 & Costa Norte do Chile \\
\hline 7112 & 341 & 2233.09 & $.27,899$ & $.70,918$ & 20 & 1749 & Costa Noted do Chile \\
\hline 1612 & 350 & 125425 & $-4,7,76$ & $.76,056$ & 10 & 3173 & Costa Sul do Chile \\
\hline
\end{tabular}

Figura 3 - Informações gerais sobre os sismos registrados pelo catálogo do NEIC.

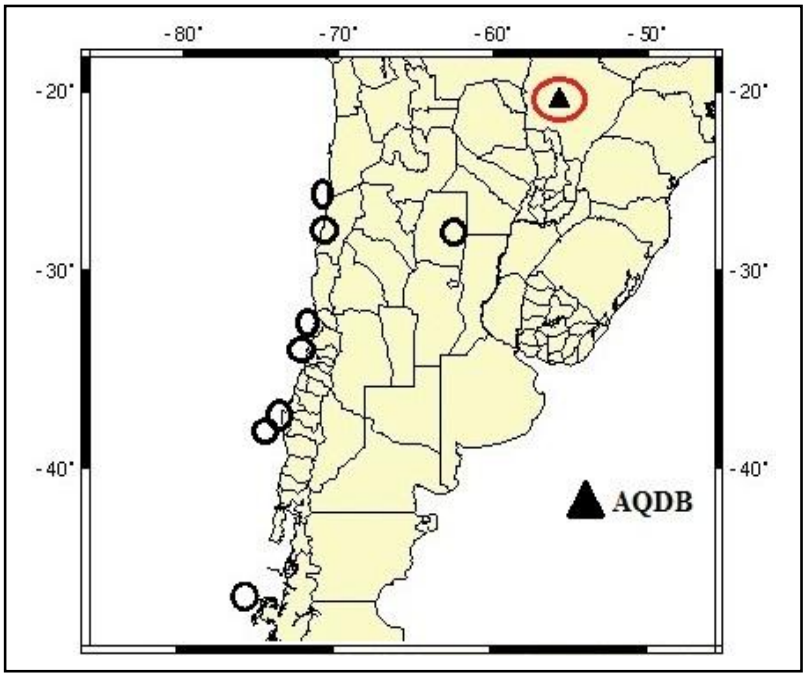

Figura 4 - Localização de alguns eventos sísmicos (epicentros) registrados pela Estação $A Q D B$.

As magnitudes $m_{b}$ e $M_{s}$ foram calculadas e comparadas em relação aos sismos catalogados pelo NEIC. Os gráficos mostram a correlação entre os dados, apresentando a porcentagem de acerto no cálculo dos sismos registrados na Estação. A magnitude $m_{b}$ teve um ajuste de curva em torno de $97.3 \%$ e a margem de erro entre os resultados obtidos teve uma média de \pm 0.16 . A magnitude $M_{s}$ teve um ajuste de curva em torno de $75.6 \%$ e a e a margem de erro teve uma média de \pm 0.2 , devido a essa diferença a dispersão entre os pontos foi maior. (Figuras 5 e 6 ).

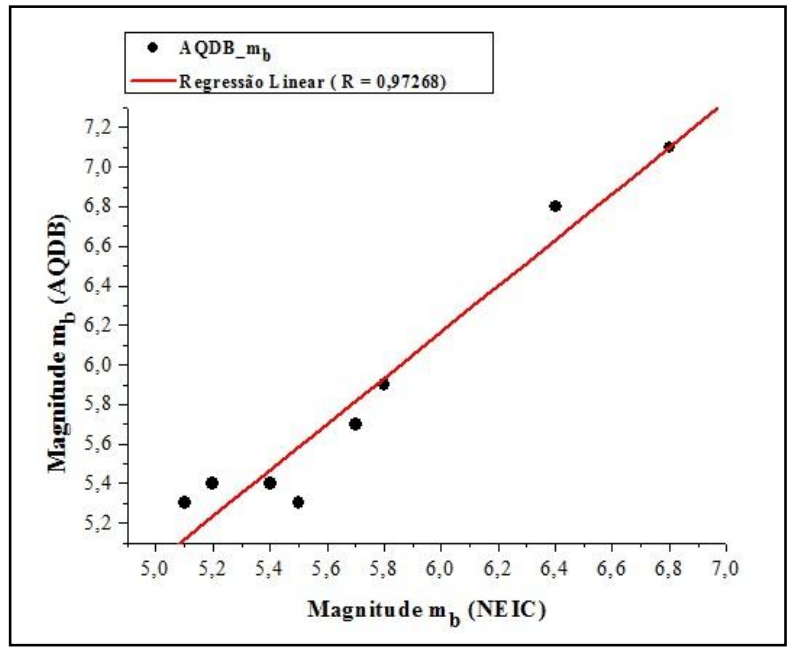

Figura 5 - Magnitude $m_{b}$ calculada $(A Q D B) \mathrm{em}$ relação ao catálogo do NEIC 


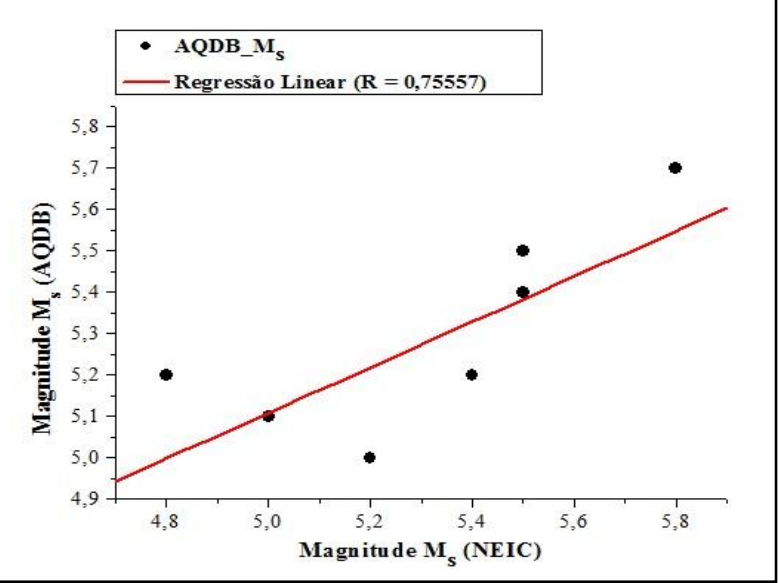

Figura 6 - Magnitude $M_{s}$ calculada (AQDB) em relação ao catálogo do NEIC

A instalação da estação AQDB no segundo semestre de 2011, através do Projeto BRASIS/Petrobrás, vem possibilitando o monitoramento da região Centro Oeste do Brasil, bem como da faixa andina. Os dados adquiridos pela Estação Sismográfica de Aquidauana apresentam dados relevantes de telessismos da Cordilheira Andina colaborando na compreensão de limites das placas litosféricas, onde predominam processos compressivos, além de possibilitar a comparação entre o NEIC e AQDB.

\section{Agradecimentos}

Agradecemos à Petrobras (Rede Temática de Geotectônica) pelo apoio ao projeto BRASIS.

\section{Referências}

Assumpção, M., et al., 2004. BLSP02: Projeto de estudo sismológico da crosta e manto superior do Brasil. São Paulo: I Simpósio Regional da Sociedade Brasileira de Geofísica.

Assumpção, M., 2012. Introdução à Sismologia. IAG/USP, São Paulo.

Brasis., 2011. Rede Sismográfica Integrada do Brasil. São Paulo: IAG/USP, 2011.

Facincani, E, M., Assumpção, M. S,. et al., 2006. Sismicidade da Região de Aquidauana - MS. Campo Grande: I Simpósio de Geotecnologias no Pantanal.

Facincani, E, M. et al., 2011. Sismicidade da Bacia do Pantanal Mato-Grossense. XIII Simpósio Nacional de Estudos Tectônicos (SNET), Campinas, São Paulo. 